\title{
RELAÇÃO ENTRE AUTOEFICÁCIA E ESTRATÉGIAS DE ENFRENTAMENTO DE USUÁRIOS ABSTINENTES DE DROGAS
}

\section{Gabriela Chaves Rodrigues}

Graduada em psicologia pela Universidade do Vale do Itajaí, Itajaí/SC, Brasil.

\section{Roberta Borghetti Alves}

Doutora em Psicologia pela Universidade Federal de Santa Catarina, Brasil. Docente do Curso de Psicologia da Universidade do Vale do Itajaí. Vice-presidente da Associação Brasileira de Psicologia Ambiental e Relações Pessoa-Ambiente, Aracaju/SE, Brasil.

\section{Priscila Rosa Martins}

Graduanda em Psicologia pela Universidade do Vale do Itajaí, Itajaí/SC, Brasil.
Autor correspondente:

Gabriela Chaves Rodrigues

gabrielachaves568@gmail.com
RESUMO: Analisar a relação entre autoeficácia e estratégias de enfrentamento em usuários abstinentes de drogas. Estudo descritivo, correlacional, com abordagem quantitativa, de campo e transversal. Aplicaram-se a Escala de Autoeficácia para Abstinência de Drogas, o Inventário de Habilidades de Enfrentamento Antecipatório para a Abstinência de Álcool e outras Drogas e um Questionário Sociodemográfico. Participaram 100 homens. Evidenciou-se que a crença de ser autoeficaz diante das emoções negativas obteve correlação positiva e significativa ao ser Assertivo na Recusa de Drogas, no Autocontrole de Emoções e na Expressão de Emoções Positivas. Já ser Assertivo na Recusa de Drogas apresentou correlação positiva e significativa com a crença de ser eficaz frente a Emoções Negativas, Situações Sociais Positivas, Preocupações com outros, e estados fisiológicos como a Síndrome de Abstinência ou Fissura. Tais relações demonstram a necessidade da utilização destes fenômenos quando se busca a abstinência de usuários de drogas.

PALAVRAS-CHAVE: Autoeficácia; Adaptação Psicológica; Transtornos Relacionados ao Uso de Substâncias.

\section{RELATIONSHIP BET WEEN SELF-EFFICACIOUSNESS AND COPING STRATEGY OF DRUG-ABSTAINING PEOPLE}

\begin{abstract}
To analyze the relationship between self-efficaciousness and coping strategies in drug-abstaining people. Descriptive, co-relational, quantitative, transversal field study. Auto-efficaciousness Scale for Drug Abstinence, Inventory of Anticipatory Coping Abilities for Alcohol and Drug Abstinence and a Socio-demographic Questionnaire, with one hundred males. Belief in auto-efficaciousness with negative emotions had a positive and significant co-relationship with regard to Assertion in Drug Refusal, Self-control in Emotions and in Expression of Positive Emotions. Assertion in Drug Refusal had a positive and significant co-relationship with belief in efficiency to cope with Negative Emotions, Positive Social Situations, Concern for Others, and physiological states such as Abstinence Syndrome or Fissure. Relationships demonstrated the need for these phenomena when drug users seek abstinence.
\end{abstract}

KEY WORDS: Self Efficacy; Adaptation Psychological; Substance-Related Disorders.

\section{INTRODUÇÃO}

O uso de substâncias de abuso lícitas e ilícitas é considerado um problema de saúde pública no Brasil e no mundo ${ }^{1}$. Aproximada- 
mente $16,9 \%$ da população adulta brasileira são fumantes, $50 \%$ da população utilizam álcool, sendo que 39\% deste grupo bebem cinco doses ou mais em um dia regular de consumo. Em relação às drogas ilícitas, 2,5\% utilizam maconha e $1,7 \%$ cocaína $^{2}$. De acordo com o United Nations Office for Drugs and Crimes ${ }^{3}$, em 2010 entre 167 a 315 milhões de pessoas, de 15 a 64 anos, utilizaram algum tipo de substância ilícita e desde 2008 houve aumento de $18 \%$ no uso de tais substâncias.

O comportamento de consumir as drogas de forma abusiva pode chegar à possível dependência, podendo contribuir para o surgimento de problemas significativos nas diversas dimensões da vida do sujeito, tornando a dependência química um fenômeno de natureza complexa e interdisciplinar ${ }^{4}$. Dentre os problemas resultantes destaca-se a prevalência de comorbidades psiquiátricas, como transtornos de humor e transtornos de ansiedade, e comorbidades clínicas, como complicações vasculares e respiratórias ${ }^{5}$, assim como redução das redes sociais, com destaque no âmbito familiar ${ }^{6}$, prejuízos financeiros e o envolvimento com a criminalidade ${ }^{7}$.

Frente aos diversos prejuízos causados pela dependência química na vida do indivíduo, o tratamento consequentemente deve ser multifatorial e interdisciplinar, integrando os diversos modelos explicativos e as suas variáveis sobre este fenômeno ${ }^{8}$. No Brasil, há duas políticas de tratamento às pessoas com dependência química, a estratégia de redução de danos, utilizada e regulamentada na saúde pública' ${ }^{9}$, a a abstinência total, utilizada em maior proporção em comunidades terapêuticas e clínicas particulares $^{10}$.

Como o presente estudo buscou-se pesquisar dois fenômenos intitulados autoeficácia e estratégias de enfrentamento em instituições que preconizam a abstinência total, focou-se em tal política. Desse modo, a política de abstinência total, assim como a política de redução de danos têm como intuito viabilizar a prevenção da recaída de usuários com uso, abuso ou dependência quími$\mathrm{Ca}^{11}$. Diversas são as variáveis que compõem o modelo de prevenção da recaída e uma delas volta-se às estratégias de enfrentamento, as quais são consideradas como um conjunto de esforços cognitivos e comportamentais para lidar com demandas externas e/ou internas específicas. Essas demandas são entendidas como situações estres- santes, pois acabam excedendo os recursos que a pessoa possui para enfrentá-las ${ }^{12}$.

$\mathrm{O}$ indivíduo ao desenvolver este repertório de estratégias de enfrentamento, além de diminuir respostas desadaptativas no manejo de situações, reduz a probabilidade do consumo de substâncias de abuso ${ }^{13-12}$. Em vista disso, as estratégias de enfrentamento do tipo antecipatório caracterizam-se como pensamentos e comportamentos direcionados para a construção de um ambiente menos suscetível às situações estressoras, antes que possam ocasionar a fissura ${ }^{8}$.

As estratégias de enfrentamento antecipatório podem dividir-se em três fatores: a "Assertividade para recusa", que envolve habilidades para recusar ofertas de substâncias. Assim como a "Expressão de sentimentos positivos", a qual está relacionada às habilidades para demonstrar sentimentos que colaboram para o estabelecimento de situações sociais menos estressoras e o "Autocontrole emocional", que envolve o manejo da raiva ${ }^{14}$.

As estratégias de enfrentamento antecipatório constituem-se como a prevenção primária da recaída no tratamento de pessoas que utilizam substâncias de abu$\mathrm{so}^{14}$. Desse modo, torna-se relevante que o indivíduo desenvolva um repertório de habilidades de enfrentamento antecipatório porque evita que situações do cotidiano acabem se transformando em situações estressoras e, consequentemente, tragam consigo menor probabilidade de ocorrer o lapso ou a recaída ${ }^{8}$.

Outra variável psicológica que integra o processo de prevenção da recaída é a autoeficácia. De acordo com Bandura ${ }^{15}$, a autoeficácia é a percepção que o indivíduo tem de si mesmo para alcançar os objetivos e metas traçados. Desta forma, quando o sujeito percebe-se capaz de realizar as ações almejadas, ele consegue ter maior controle sobre o seu funcionamento ${ }^{16}$.

Ademais, a autoeficácia é um construto que depende do contexto onde está sendo exercida, sendo necessário analisar as condições específicas em que o indivíduo irá utilizar a autoeficácia ${ }^{15}$. Dentro deste contexto, ocorrem eventos ambientais que influenciam a avaliação cognitiva feita pelo indivíduo acerca da autoeficácia, pois algumas situações irão exigir maior habilidade e desempenho do indivíduo ${ }^{15}$. De fato, os indivíduos que são tomados por dúvidas sobre sua eficácia tendem a visualizar 
cenários de falha, já os indivíduos que possuem elevado senso de eficácia conseguem visualizar cenários de sucesso, o qual fornece suporte para o desempenho ${ }^{17}$.

A função de uma elevada autoeficácia em pessoas com dependência química pode influenciar comportamentos futuros, já que há a expectativa do indivíduo de não consumir substâncias de abuso e a crença de que é capaz de fazê-lo ${ }^{18}$. Nesta perspectiva, a autoeficácia voltada para a abstinência caracteriza-se como a crença do indivíduo na própria capacidade de resistir às substâncias de abuso em situações de risco ${ }^{19}$. Em relação ao contexto, é possível avaliar a autoeficácia para abstinência de acordo com as situações de risco baseadas em quatro domínios: emoções negativas, social-positivo, preocupações e abstinência-fissura ${ }^{20}$.

No que se refere à autoeficácia frente as emoções negativas, consiste quando o usuário percebe-se capaz de lidar com emoções como a depressão, tristeza, tensão, tédio, frustração, raiva e descrédito. Essas emoções negativas são vivenciadas em situações consideradas desagradáveis ou aversivas, como brigas, dificuldades de emprego e financeiras, ou quando ocorre alguma adversidade pessoal. Já na dimensão social/positivo, o usuário avalia-se competente em lidar com emoções positivas desencadeadas em situações sociais favoráveis ao uso. Essas emoções positivas consistem em estar relaxado, eufórico, corajoso e ter excesso de confiança em situações de confrontamento da droga. Em relação à autoeficácia diante de preocupações, refere-se à capacidade do usuário de enfrentar dores físicas, o cansaço, sonhar com o uso de drogas ou preocupar-se com outras pessoas. $\mathrm{Na}$ dimensão abstinência/fissura, o usuário utiliza a autoeficácia para lidar com estados físicos associados ao uso prévio das substâncias de abuso, como a síndrome de abstinência ou a fissura. Esses estados físicos referem-se à necessidade e à vontade do corpo de utilizar novamente as substâncias de abuso ${ }^{20}$.

Diante do exposto, o uso de estratégias de enfrentamento antecipatório, em situações de risco, pode proporcionar às pessoas com dependência química a prevenção ou o manejo da fissura, promovendo o uso de comportamentos mais adaptativos ${ }^{14}$. Da mesma forma, a autoeficácia para abstinência também mostra-se um importante elemento no processo de mudança de comportamentos relacionados ao consumo de substâncias de abuso ${ }^{20}$. Frente a estas ponderações, o presente estudo buscou analisar a relação entre a autoeficácia e as estratégias de enfrentamento antecipatório em usuários abstinentes de substâncias de abuso.

\section{METODOLOGIA}

\section{NATUREZA DA PESQUISA}

O presente estudo caracterizou-se como uma pesquisa descritiva correlacional, pois pretendeu descrever e correlacionar as características dos fenômenos estratégias de enfrentamento antecipatório e autoeficácia para abstinência ${ }^{21,22}$. Também classificou-se como pesquisa de campo e transversal, já que a coleta de dados procedeu junto de pessoas ${ }^{23}$ e ocorreu em um determinado período com grupos etários ${ }^{24}$.

Quanto à abordagem da pesquisa esta foi quantitativa, em razão de utilizar procedimentos estruturados e instrumentos formais para coletar os dados da amostra, enfatizando a objetividade ${ }^{25}$. E aplicada, pois, objetiva gerar conhecimentos para aplicação prática, dirigidos à solução de problemas específicos ${ }^{26}$.

\section{PARTICIPANTES DA PESQUISA}

Participaram do estudo 100 homens abstinentes de substâncias de abuso. A coleta de dados ocorreu em cinco comunidades terapêuticas localizadas na região do Vale do Itajaí. Os critérios de inclusão compreendiam ser do sexo masculino, com idade superior a 18 anos e estar no mínimo 14 dias abstinente da(s) substância (s) psicoativa (s) que fazia uso. O período de abstinência escolhido justifica-se pelos usuários das comunidades terapêuticas estarem em processo de desintoxicação.

Conforme a Tabela 1, que aborda o perfil sociodemográfico dos participantes, a média de idade dos participantes foi de 38 anos, com desvio padrão (DP) de 9,85 anos. Quanto ao tempo de abstinência, obteve-se uma média de cinco meses $(\mathrm{DP}=19)$. No que consiste à quantidade de filhos, $71 \%$ dos participantes possuíam filhos, sendo que a média foi de um filho por participante 
$(\mathrm{DP}=1,31)$. Já a quantidade de internações atingiu uma média de três vezes $(\mathrm{DP}=2,58)$. E o tempo médio em que os usuários estão nas comunidades foi de três meses $(\mathrm{DP}=1,84)$.

Entre todos os entrevistados, $47 \%$ dos participantes obtiveram o ensino médio, seguido de $46 \%$ com ensino fundamental. No que se refere ao estado civil, de acordo com a opinião dos participantes, $57 \%$ eram solteiros. Quanto à religião, 57\% dos participantes consideravam-se evangélicos protestantes; neste caso, quatro das cinco comunidades onde foram realizadas as coletas eram de origem evangélica. Em relação à profissão, 50\% pertenciam à categoria Produção de Bens e Serviços Industriais, na qual se encaixam as seguintes profissões (auxiliar de sondagem, construção civil, construtor, eletricista, latoeiro, operador de máquina, pedreiro, dentre outros). Referente à substância que levou os participantes a procurar pelo tratamento, $68 \%$ faziam uso de crack ou cocaína, e $82 \%$ tiveram a iniciativa para ir à Comunidade Terapêutica procurar tratamento.

Tabela 1. Perfil sociodemográfico dos participantes

\begin{tabular}{lccc} 
& \multicolumn{3}{c}{ (Continua) } \\
\hline \multirow{2}{*}{ Variáveis } & Participantes $(\mathrm{n}=100)$ \\
\cline { 2 - 4 } & $\mathrm{N}$ & $\mathrm{M} / \mathrm{DP}$ & $\%$ \\
\hline Idade (anos) & 100 & $38 / 9,85$ & 100 \\
\hline Tempo de abstinência & & & \\
\hline$<=6$ meses & 94 & $5 / 19$ & 6 \\
$>6$ meses & 6 & & 94 \\
\hline Quantidade de filhos & & & \\
\hline Nenhum & 29 & & 29 \\
1 filho & 28 & $1 / 1,31$ & 28 \\
Mais de 1 filho & 43 & & 43 \\
\hline Quantidade de internações & & & \\
\hline$<=3$ & 82 & $3 / 2,58$ & 82 \\
$>3$ & 12 & & 12 \\
\hline Tempo de internação & & & \\
\hline$<=3$ meses & 59 & 31,84 \\
\hline 3 meses & 41 & & \\
\hline Escolaridade & & & \\
\hline & & & \\
\hline
\end{tabular}

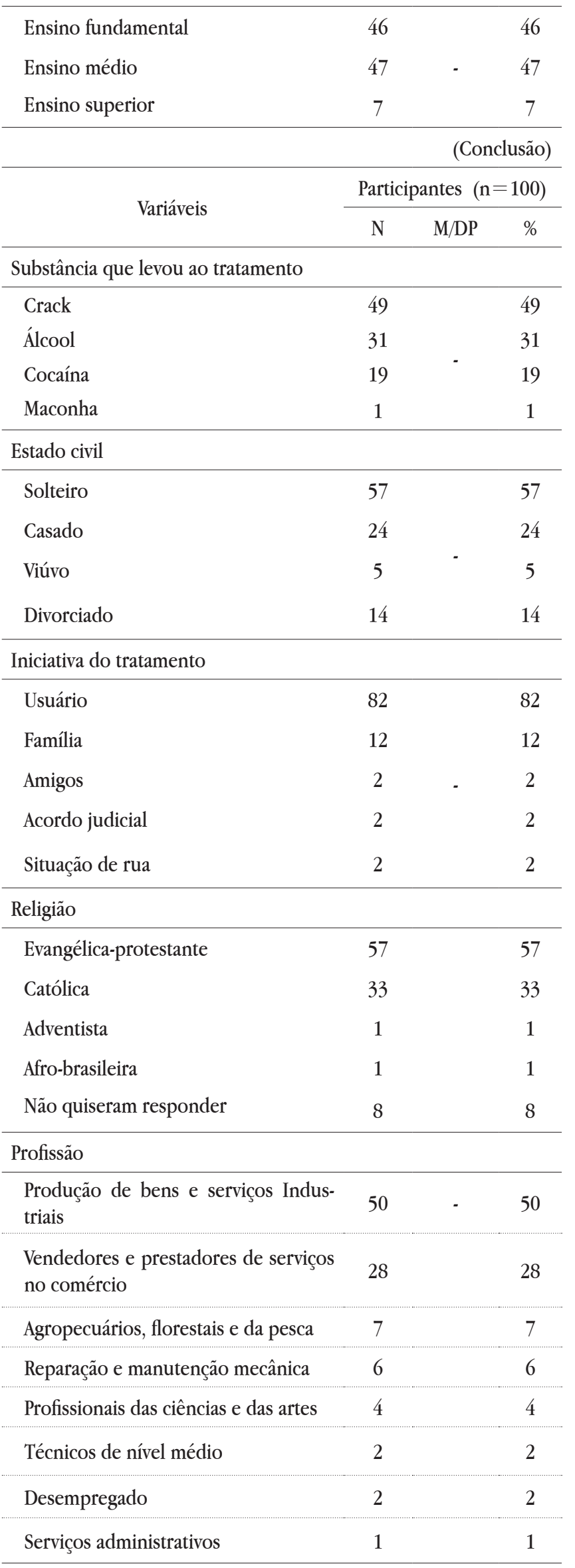




\section{INSTRUMENTOS DE COLETA DE DADOS}

Para a coleta de dados, foi aplicado um questionário sociodemográfico, construído pelas próprias autoras, as quais englobaram perguntas voltadas aos dados pessoais (a idade, escolaridade, estado civil, número de filhos, religião, profissão) e a (s) substância (s) de abuso (tempo sem uso, substância(s) que utilizou, substância que levou à comunidade terapêutica). Além disso, perguntou-se acerca da internação em comunidade terapêutica (quantas vezes o usuário esteve em comunidades terapêuticas, tempo em que está na comunidade e o motivo o qual fez ele procurar a comunidade).

No que se refere à avaliação do fenômeno estratégias de enfrentamento antecipatório, foi utilizado o inventário desenvolvido por Sá ${ }^{14}$, o qual se intitula Inventário de Habilidades de Enfrentamento Antecipatório (IDHEA-AD), em que possui duas versões, sendo uma destinada para usuários de álcool (A) e outra para as demais drogas (D). Os comportamentos avaliados nos itens são idênticos e diferenciando-se apenas na sua redação. O inventário contém 30 itens distribuídos em três fatores: Assertividade para Recusa (14 itens), Expressão de Sentimentos Positivos (8 itens) e Autocontrole Emocional (8 itens). Os itens dividem-se em uma escala tipo Likert de 4 pontos, dos quais 0 ponto significa "nunca", 1 ponto significa "poucas vezes", 2 pontos significam "muitas vezes" e 3 pontos significam "sempre".

$\mathrm{O}$ fator Assertividade para Recusa, envolve assertividade e planejamento para situações de alto risco de consumo de substâncias. Já o fator Expressão de Sentimento Positivo, refere-se às habilidades para demonstrar sentimentos que colaboram para o estabelecimento de situações sociais menos estressoras. E o fator Autocontrole Emocional envolve, principalmente, o manejo da raiva. $\mathrm{O}$ inventário apresenta evidências de validade de consistência interna e precisão $(\alpha=0.88)$.

Já o construto autoeficácia foi mensurado pela escala de autoeficácia para Abstinência de Drogas (EAAD), traduzida e adaptada para língua portuguesa por Freire ${ }^{20}$ da escala original, desenvolvida por DiClemente et al. ${ }^{27}$, intitulada Alcohol Abstinence Self-efficacy Scale (AASE). O instrumento contém 24 itens que se distribuem em quatro fatores, emoções negativas, social/positivo, preocupações e abstinência/fissura. As alternativas são classificadas em uma escala Likert de 5 pontos, de 1 - "Nada seguro" a 5 - "Extremamente seguro de não usar drogas". A escala possui evidências de validade de consistência interna, relações externas e precisão $(\alpha=0,92)$.

O fator emoções negativas consiste quando o usuário percebe-se capaz de lidar com emoções como a depressão, tristeza, tensão, tédio, frustração, raiva e descrédito. O fator social/positivo diz respeito em lidar com emoções positivas desencadeadas em situações sociais favoráveis ao uso, como estar relaxado, eufórico, corajoso e ter excesso de confiança. Em relação ao fator preocupações, refere-se à capacidade do usuário de enfrentar dores físicas, o cansaço, sonhar com o uso de drogas ou preocupar-se com outras pessoas. E o fator abstinência/ fissura é quando o sujeito percebe-se capaz de lidar com estados físicos associados ao uso prévio das substâncias de abuso, como a síndrome de abstinência ou a fissura.

\section{PROCEDIMENTOS PARA A COLETA DOS DADOS}

Inicialmente, foi realizado o primeiro contato com as cinco comunidades terapêuticas onde se explicou sobre a temática e objetivos da pesquisa. Após os esclarecimentos, foi pedido autorização para a coleta de dados nos locais mencionados. Mediante o aceite, o projeto foi submetido ao Comitê de Ética da Universidade do Vale do Itajaí (Univali). Para o desenvolvimento dessa pesquisa, a mesma teve como norte o que preconiza a resolução no 466/2012 do Conselho Nacional de Saúde ${ }^{28}$, de modo a buscar atender às normas voltadas às questões éticas e de respeito em relação às pesquisas realizadas com seres humanos. Tendo a autorização do Comitê de Ética, por meio do número do parecer 2.742.659, foi possível voltar às comunidades terapêuticas, onde os usuários foram convidados para participarem da pesquisa. Nesse contato, foi explicado o Termo de Consentimento Livre e Esclarecido (TCLE), de modo a destacar que pesquisa buscaria garantir o anonimato, além de possuir caráter voluntário, bem como a possibilidade de desistência a qualquer momento da pesquisa. Explicou-se sobre a 
natureza da pesquisa, justificativa, objetivos e riscos. Foi garantido que o participante teria o tempo necessário para tomar a decisão autônoma acerca de sua participação ou não no estudo, assim como buscar respeitar sua singularidade.

A partir do aceite, ocorreu a aplicação dos instrumentos individualmente, em um ambiente privado, o qual estavam apenas o participante e a pesquisadora, respeitando o anonimato e as informações relatadas pelos sujeitos. Entre os instrumentos aplicados, o primeiro foi o questionário sociodemográfico, seguido do Inventário de Habilidades de Enfrentamento Antecipatório (IDHEA) e da escala de Autoeficácia para Abstinência de Drogas (EAAD). Posteriormente, os dados foram tabulados e analisados estatisticamente. Assim que se findaram a análise e o trabalho, foi realizada a devolutiva nas comunidades terapêuticas, onde os usuários foram convidados a comparecerem a estes locais para receberem a devolutiva.

\section{PROCEDIMENTOS PARA A ANÁLISE DOS DADOS}

Os dados foram tabulados por meio do software Statistical Package for the Social Siences (SPSS) versão 22.0. Mediante a tabulação dos dados, realizou-se a análise por meio da estatística descritiva e correlacional. Pela normalidade dos resultados, utilizou-se o teste de correlação de Pearson para análise de variáveis numéricas e para os testes de diferenças de médias, foi aplicado o teste T.

\section{RESULTADOS}

A Tabela 2 apresenta as correlações de Pearson entre os fatores da escala de Autoeficácia para Abstinência de Drogas (EAAD) e do Inventário de Habilidades de Enfrentamento para Abstinência de Álcool e outras Drogas (IDHEA-AD). Observa-se que o fator emoções Negativas obteve correlação significativa $(p<0,01)$ com os fatores Assertividade para Recusa $(\mathrm{r}=0,44)$ e Autocontrole Emocional $(r=0,34)$, e embora baixa também demonstrou correlação significativa $(p<0,05)$ com o fator Expressão de Sentimentos Positivos $(r=0,20)$. No que se refere ao fator Assertividade para Recusa, apresentou correlação significativa $(p<0,01)$ com os fatores emoções negativas, como já mencionado, social/positivo $(r=0,32)$, preocupações $(r=0,26)$ e abstinência/fissura $(r=0,27)$.

Já as variáveis idade, tempo de abstinência, quantidade de internação e tempo de internação não apresentaram correlação significativa com os fatores da autoeficácia e das estratégias de enfrentamento. Embora não seja o objetivo desse artigo, salienta-se que a variável idade demonstrou correlação significativa $(p<0,05)$ com a variável tempo de internação $(r=0,24)$. Por sua vez, a variável tempo de abstinência exibiu correlação significativa $(\mathrm{p}<0,01)$ com a quantidade de internação $(\mathrm{r}=0.39)$.

Tabela 2. Correlação entre os fatores da autoeficácia (EAAD) e fatores das estratégias de enfrentamento (IDHEA-AD)

\begin{tabular}{|l|c|c|c|}
\hline \multirow{2}{*}{$\begin{array}{c}\text { Fatores da autoeficácia } \\
\text { (EAAD) }\end{array}$} & $\begin{array}{c}\text { Fatores das estratégias de enfrentamen- } \\
\text { to (IDHEA-AD) }\end{array}$ \\
\cline { 2 - 4 } & $\begin{array}{c}\text { Assertivi- } \\
\text { dade para } \\
\text { recusa }\end{array}$ & $\begin{array}{c}\text { Expressão } \\
\text { de sen- } \\
\text { timento } \\
\text { positivo }\end{array}$ & $\begin{array}{c}\text { Autocontrole } \\
\text { emocional }\end{array}$ \\
\hline Emoções negativas & $0,44^{* *}$ & $0,20 *$ & $0,34^{* *}$ \\
Social/positivo & $0,32^{* *}$ & 0,11 & 0,17 \\
Preocupações & $0,26 * *$ & 0,05 & 0,17 \\
Abstinência & $0,27 * *$ & 0,14 & 0,16 \\
\hline /fissura & & & \\
\hline
\end{tabular}

Nota: $* \mathrm{p}<0,05 ; * * \mathrm{p}<0,01$

Já a Tabela 3 apresenta as médias e os desvios-padrão para cada fator da autoeficácia (EAAD) e das estratégias de enfrentamento (IDHEA-AD), por ter ou não ter religião, a substância álcool ou crack/cocaína, o nível de significância observado na comparação das médias (teste t) e os índices de consistência interna dos instrumentos. Verificou-se diferença estaticamente significativa $(p<0,01)$ para as variáveis não ter religião ou ter religião quando se trata do fator autocontrole emocional para estratégias de enfrentamento antecipatório. Observou-se, ainda, diferença estatisticamente significativa $(p<0,05)$ para as variáveis álcool ou crack/cocaína no fator assertividade para recusa para estratégias de enfrentamento. 
Tabela 3. Médias e desvios-padrão dos fatores nos dois instrumentos, conforme religião, tipo de substância e nível de significância da comparação das médias (test t)

\begin{tabular}{|c|c|c|c|c|c|c|c|c|c|c|c|c|}
\hline \multirow[t]{2}{*}{ Fatores } & \multicolumn{2}{|c|}{$\begin{array}{l}\text { Não ter religião } \\
\qquad(\mathrm{n}=8)\end{array}$} & \multicolumn{2}{|c|}{$\begin{array}{l}\text { Ter religião } \\
(\mathrm{n}=92)\end{array}$} & \multirow[b]{2}{*}{$\mathrm{t}$} & \multicolumn{3}{|c|}{ Álcool $(n=31)$} & \multicolumn{2}{|c|}{$\begin{array}{c}\text { Crack/cocaína } \\
(n=69)\end{array}$} & \multirow[b]{2}{*}{$\mathrm{t}$} & \multirow[b]{2}{*}{$\mathrm{p}$} \\
\hline & Média & $\mathrm{dp}$ & Média & $\mathrm{dp}$ & & $\mathrm{p}$ & Média & $\mathrm{dp}$ & Média & $\mathrm{dp}$ & & \\
\hline \multicolumn{13}{|l|}{ Autoeficácia (EAAD) } \\
\hline Emoções negativas & 20,6 & 6,8 & 21,2 & 6,7 & $-0,24$ & ns $(0,8)$ & 20,1 & 7,1 & 21,6 & 6,4 & $-1,03$ & ns $(0,3)$ \\
\hline Social/positivo & 22,4 & 8,2 & 21,5 & 6,9 & 0,34 & ns $(0,7)$ & 20,5 & 8,0 & 22,0 & 6,4 & $-1,00$ & ns $(0,3)$ \\
\hline Preocupações & 19,5 & 3,4 & 17,0 & 4,3 & 1,54 & ns $(0,12)$ & 16,8 & 4,9 & 17,4 & 4,0 & $-0,63$ & ns $(0,5)$ \\
\hline Abstinência/fissura & 15,9 & 5,2 & 15,0 & 4,9 & 0,46 & ns $(0,6)$ & 14,4 & 5,7 & 15,4 & 4,5 & $-0,95$ & ns $(0,3)$ \\
\hline \multicolumn{13}{|c|}{ Estratégias de enfrentamento (IDHEA-AD) } \\
\hline Assertividade para recusa & 28,2 & 10,3 & 30,9 & 9,5 & $-0,76$ & ns $(0,44)$ & 27,6 & 9,4 & 32,1 & 9,3 & $-2,21$ & $0,02 *$ \\
\hline $\begin{array}{l}\text { Expressão de sentimentos } \\
\text { positivos }\end{array}$ & 14,4 & 4,9 & 17,7 & 5,5 & $-1,62$ & ns $(0,10)$ & 17,9 & 4,7 & 17,1 & 5,9 & 0,57 & ns $(0,5)$ \\
\hline Autocontrole emocional & 12,6 & 4,5 & 17,0 & 4,1 & $-2,92$ & $0,00 * *$ & 16,6 & 3,9 & 16,8 & 4,5 & $-0,23$ & ns $(0,8)$ \\
\hline
\end{tabular}

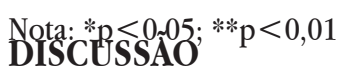

O objetivo do presente estudo foi analisar a relação entre a autoeficácia e as estratégias de enfrentamento antecipatório em usuários abstinentes de substâncias de abuso. Assim sendo, verificou-se nesta pesquisa que quanto mais o indivíduo acredita ser eficaz para lidar com emoções negativas, mais ele consegue ser assertivo na recusa de substâncias de abuso, ter autocontrole emocional e expressar sentimentos que colaboram para o estabelecimento de situações sociais menos estressoras. Estes resultados foram ao encontro do estudo de Sá ${ }^{14}$, no que consiste a relação positiva e significativa das variáveis autoeficácia e estratégias de enfrentamento. No entanto, acerca da magnitude do fator assertividade para recusa, Sá ${ }^{14}$ obteve correlação forte. Hipotetiza-se que a magnitude dessa correlação foi encontrada pela amostra deste autor estar em tratamento ambulatorial, cumprindo até 12 horas de tratamento. Neste caso, sugere-se que o tratamento ambulatorial permite o desenvolvimento de um repertório de enfrentamento mais elaborado nos sujeitos, por caracterizar-se em um ambiente menos controlado. Já na comunidade terapêutica, ambiente pesquisado do presente estudo, os sujeitos teriam menos oportunidades de desenvolver o repertório de estratégias de enfrentamento, pois caracteriza-se como um ambiente isolado, onde se evitam exposições de situações de risco ${ }^{13}$.
Ainda, os resultados apontaram que quanto mais o usuário percebe-se capaz de lidar com situações sociais desencadeadoras do uso de substâncias de abuso, mais o usuário consegue ser assertivo na recusa tais substâncias. O mesmo ocorre quando o usuário enfrenta situações que lhe causam preocupações ou quando sente uma necessidade física de consumir a droga, maior será o uso de estratégias de recusa das substâncias de abuso. Estes resultados vieram ao encontro do estudo de Litt, Kadden e Petry ${ }^{30}$, os quais encontraram em um grupo de pessoas que estavam em abstinência total de uso de drogas, o aumento tanto na expectativa de eficácia quanto no desenvolvimento de habilidades de enfrentamento.

Resultados similares foram encontrados no estudo de Rose et al. ${ }^{31}$, os quais avaliaram pessoas em tratamento em uma clínica com foco na abstinência. Os resultados do estudo indicaram melhorias na autoeficácia e na eficácia dos comportamentos de enfrentamento para evitar o uso de álcool. Além dos autores supracitados, Coelho ${ }^{32}$ observou o aumento de habilidades de enfrentamento e da autoeficácia para abstinência, imediatamente após o término e após três meses de acompanhamento do Treinamento de Habilidades Sociais e de Enfrentamento (THSE). O TSHE baseou-se no modelo de Prevenção de Recaída, com o intuito de desenvolver 
as habilidades de enfrentamento assim como fortalecer a autoeficácia de pessoas que faziam uso de crack.

De acordo com o estudo de Tenhula, Bennett e Kinnaman33, foi possível verificar o uso de estratégias de enfrentamento no manejo de emoções negativas. Uma vez que, por meio de um tratamento comportamental para doenças graves e persistentes, observou-se o planejamento de estratégias de enfrentamento para situações de alto risco, as quais o sujeito sentia emoções negativas, como tédio, depressão ou estresse. Desta forma, quanto mais o sujeito conseguia aplicar tais estratégias e perceber a eficácia dos resultados, mais o sujeito sentia-se motivado e capaz de alcançar a manutenção da abstinência.

No que consiste às variáveis idade, tempo de abstinência, quantidade de internação e tempo de internação não demonstraram correlações significativas com os fatores da Autoeficácia e das Estratégias de Enfrentamento. Já no que consiste a variável idade, esta demonstrou que quanto maior for a idade mais tempo a pessoa tende a ficar internada $(r=0,24)$. Já quanto mais tempo internada, maior será quantidade de internações que a pessoa tende a ter, ou seja, quanto mais tempo ela permanece na comunidade terapêutica, mais vezes ele tende a procurar a comunidade terapêutica para permanecer internado $(r=0.39)$. No Brasil, a internação ainda é considerada a principal forma de intervenção às pessoas com dependência química. A entrada e permanência nas comunidades terapêuticas se dá por situações de vulnerabilidade física, emocional e social do usuário33. Alguns exemplos são a situação de rua, a debilidade física e o rompimento de laços familiares, fazendo com que os usuários busquem o serviço que possa melhor atender suas demandas imediatas34, de modo a corroborar para com os dados evidenciados.

Ademais, os resultados supracitados foram corroborados com os estudos de Araújo et al.35 e Araújo et al.36, os quais não encontraram correlação significativa entre o número de internações, idade, tempo de abstinência e estratégias de enfrentamento para o manejo da fissura. Contudo, no estudo de Dolan, Martin e Rohsenow37, foi possível encontrar correlação significativa entre idade avançada, menos anos de uso de drogas e autoeficácia. Assim como, no estudo de Martin et al.38, foi possível encontrar correlação significativa entre a autoe- ficácia e o tempo de abstinência em pessoas que faziam uso abusivo de álcool.

No que se refere aos resultados do teste, pessoas que utilizam a substância crack/cocaína apresentaram nesta pesquisa maior assertividade para recusa da droga do que pessoas que fazem uso do álcool. Estes resultados vieram ao encontro ao estudo de Sá e Del Prette39, os quais identificaram que quanto maior o envolvimento com crack, maiores as habilidades para discordar de autoridade e encerrar conversação. Diante do exposto, é possível que a propensão à impulsividade, à tomada de risco e à necessidade de iniciar interações pela ilegalidade da droga sejam responsáveis pelo resultado encontrado40-39.

Outra diferença estatisticamente significativa diz respeito ao fator autocontrole emocional e a categoria religião. Este estudo demonstrou que as pessoas que dizem ter uma religião utilizam mais estratégias de enfrentamento voltadas a ter autocontrole emocional do que pessoas que não têm religião. O estudo de Felipe, Carvalho e Andrade41 confirma esta evidência, os quais encontraram correlação indireta entre religiosidade e o uso de substâncias, sendo esta correlação mediada pelo autocontrole do sujeito. Desse modo, acredita-se que a religiosidade promove nesses sujeitos um auxílio na dimensão emocional que contribui para a inibição do uso de substâncias de abuso.

\section{CONCLUSÃO}

Os resultados observados indicaram correlações significativas entre os fatores das estratégias de enfrentamento antecipatório (IDHEA-AD) e da autoeficácia para abstinência (EAAD) de modo a evidenciar a necessidade do diálogo sobre estes fenômenos em espaços que preconizam a abstinência total. Outro aspecto a ser salientado é a importância de o usuário ter uma crença religiosa, pois esta variável demonstrou-se importante para a elaboração de estratégias voltadas ao autocontrole emocional. Além disso, destaca-se que usuários de crack e cocaína demonstraram maior assertividade para a recusa da droga.

Nesse sentido, a presente pesquisa pode contri- 
buir para evidenciar as principais estratégias de enfrentamento antecipatório e os contextos que circundam a autoeficácia para abstinência. A partir destes resultados, os profissionais de saúde poderão nortear estratégias de tratamento e reabilitação psicossocial. Como exemplo, sugere-se que em terapias grupais, as pessoas que fizeram uso de substâncias sejam incentivadas a relatarem as estratégias que adotam para recusar a droga. Esses relatos podem incentivar os demais usuários a utilizarem tais recursos. No entanto, recomenda-se que outras pesquisas sejam realizadas para confirmar e/ou refutar os resultados mencionados. Caso confirmem-se os resultados encontrados, indica-se que sejam investigados os motivos pelos quais homens que fazem uso de crack e cocaína tenham mais assertividade para recusa do que os usuários de álcool. Ainda, sugere-se que sejam investigadas as razões responsáveis por levar esses usuários a alcançar a assertividade para recusa pelo enfrentamento das emoções negativas.

Quanto à limitação da pesquisa salienta-se a utilização de poucas variáveis para verificar a relação entre as mesmas. Desta forma, sugere-se para futuras pesquisas o uso de instrumentos que avaliem outras variáveis, como o questionário University of Rhode Island Change Assessment Questionnaire (URICA), adaptado na população brasileira por Szupszynski e Oliveira42, o qual avalia os estágios de motivação para mudança. Salienta-se também a escala de Gravidade de Dependência, elaborada por McLellan et al.43, na qual avalia os prejuízos causados pelo abuso de substâncias de abuso, o teste de dependência à nicotina de Fagerström (FTND), validado no Brasil por Carmo e Pueyo44, o qual avalia o grau de dependência de nicotina e a escala de tentação para uso de drogas27, responsável por avaliar a tentação para usar determinadas substâncias de abuso. Por fim, salienta-se que embora seja um estudo exploratório foi possível trazer subsídios para práticas dos profissionais de saúde, assim como refletir quais variáveis possuem relação quando se analisam os construtos autoeficácia e estratégias de enfrentamento frente às substâncias de abuso.

\section{REFERÊNCIAS}

1. Bolonheis-Ramos RCM, Boarini ML. Comunidades terapêuticas: "novas" perspectivas e propostas higienistas. Hist cienc saude-Manguinhos. [internet] 2015 [ acesso em 2019 Fev 11]; 22(4): 1231-1248. Disponível em: http://www. scielo.br/scielo.php?script $=$ sci_arttext $\&$ pi$\mathrm{d}=$ S0104-59702015000401231\&lng $=\mathrm{pt \& t} \operatorname{lng}=\mathrm{pt}$ doi: $10.1590 / \mathrm{S} 0104-59702015000400005$

2. Laranjeira R, organizador. Segundo Levantamento Nacional de Álcool e Drogas - (LENAD). Instituto Nacional de Ciência e Tecnologia para Políticas Públicas de Álcool e Outras Drogas (INPAD). São Paulo; 2012.

3. United Nations Office Drugs and Crime. World drug report. UNODC: 2013; 151.

4. American Psychiatric Association. DSM-5: manual diagnóstico e estatístico de transtornos mentais. 5nd ed. Porto Alegre: Artmed; 2014. 992.

5. Capistrano FC, Ferreira ACZ, Silva TL, Kalinke LP, Maftum, MA. Perfil sociodemográfico e clínico de dependentes químicos em tratamento: análise de prontuários. Esc Anna Nery Rev Enferm. [internet] 2013 [ acesso em 2019 Fev 11]; 17 (2): 234-241. Disponível em: http://www. scielo.br/scielo.php?script $=$ sci_arttext $\&$ pi$\mathrm{d}=$ S1414-81452013000200005\&lng $=\mathrm{pt} \& \operatorname{tlng}=\mathrm{pt}$ doi: $10.1590 / \$ 1414-81452013000200005$

6. Cavalcante LP, Falcão RST, Lima HP, Marinho AM, Macedo JQ, Braga VAB. Rede de apoio social ao dependente químico: ecomapa como instrumental na assistência em saúde. Rev Rene. [internet] 2012 [acesso em 2019 Fev 15]; 13 (2): 321-331. Disponível em: http://periodicos.ufc.br/rene/article/view/3920 doi: 10.15253/rev\%20rene.v13i2.3920

7. Nimtz MA, Tavares AMF, Maftum MA, Ferreira ACZ, Capistrano FC. Impactos legais e no trabalho na vida do dependente químico. SMAD Rev eletrônica saúde mental alcool drog. [internet] 2016 [acesso em 2019 Fev 11]; 12(2): 68-74. Disponível em: http:// www.revistas.usp.br/smad/article/view/120766 doi: 


\subsection{6/issn.1806-6976.v12i2p65-74}

8. Donovan DM. Assessment of addictive behaviors for relapse prevention. In: Donovan, DM, Marlatt AG, editores. Assessment of addictive behaviors. New York: The Guilford Press; 2013. p. 1-48.

9. Brasil. Ministério da Saúde. Secretaria Executiva. Coordenação Nacional de DST e AIDS. A política do Ministério da Saúde para atenção integral a usuários de álcool e outras drogas. Ministério da Saúde: 2003; 60 .

10. De Leon G. A comunidade terapêutica: teoria, modelo e método. São Paulo: Edições Loyola; 2003.

11. Coelho C, Pérez-Ramos AMQ. Abstinência e a redução de danos no processo de recuperação dos dependentes de substância psicoativas. Bol Acad Paul Psicol. 2008; 28(1): 79-86.

12. Lazarus RS, Folkman S. Stress, appraisal, and coping. New York: Springer Publishing Company; 1984.

13. Coelho LRM, Sá LGC, Oliveira MS. Estratégias e Habilidades de Enfrentamento de Usuários de Crack em Tratamento. Rev psicol IMED. [internet] 2015 [acesso em 2019 Fev 15]; 7(2): 99-109. Recuperado de http:// www.mpdft.mp.br/saude/images/saude_mental/Estrategias_habilidades.pdf doi: 10.18256/2175-5027/ psico-imed.v7n2p99-109

14. Sá, LGC. Propriedades psicométricas do inventário de habilidades de enfrentamento para a abstinência de álcool e outras drogas (IDHEA-AD) [tese]. São Carlos: Universidade Federal de São Carlos; 2013. p. 136.

15. Bandura A. Self-efficacy: toward a unifying theory of behavioral change. Psychol rev. [internet] 1977 [acesso em 2018 Dez 5]; 84(2), 191-215. Disponível em: https://psycnet.apa.org/record/1977-25733-001 doi: 10.1037/0033-295X.84.2.191

16. Bandura A. Human agency in social cognitive theory. Am psychol. [internet] 1989 [acesso em 2018 Dez 5]; 44(9), 1175-1184. Disponível em: https://psycnet.apa.org/doiLanding?doi $=10.1037 \%$ -
2F0003-066X.44.9.1175 doi: $10.1037 / 0003-$ 066X.44.9.1175

17. Bandura A. Self-efficacy. In: Ramachaudran VS, editor. Encyclopedia of human behavior. New York: Academic Press; 1994. p. 71-81.

18. 18. Crouch TB, Diclemente CC, Pitts SC. End-of-Treatment Abstinence Self-Efficacy, Behavioral Processes of Change, and Posttreatment Drinking Outcomes in Project MATCH. Psychol addict behav. [internet] 2015 [acesso em 2018 Dez 5]; 29 (3), 706-715. Disponível em: https://psycnet.apa.org/doiLanding?doi $=10.1037 \% 2$ Fadb0000086 doi: $10.1037 /$ adb0000086

19. Diclemente CC, Fairhurst S, Piotrowski N. The role of self-efficacy in the addictive behaviors. In: Maddux J, editor. Self-efficacy, adaptation and adjustment: theory, research and application. New York: Plenum Press; 1995. p. 109-141.

20. Freire SD. (2009). Evidências de validade da escala de auto-eficácia para abstinência de drogas (EAAD) e da escala de tentação para uso de drogas (ESTUD) em dependentes de cocaína e crack internados [dissertação]. Porto Alegre: Pontifícia Universidade Católica do Rio Grande do Sul (PUCRS), Faculdade de Psicologia; 2009. p. 119.

21. Gil AC. Como elaborar projetos de pesquisa. 4nd ed. São Paulo: Atlas; 2002. Como classificar as pesquisas; p. 41-57.

22. Triviños ANS. Introdução à pesquisa em ciências sociais: a pesquisa qualitativa em educação. São Paulo: Atlas; 1987.

23. Fonseca JJS. (2002). Apostila de Metodologia da Pesquisa Científica. Fortaleza: Universidade estadual do Ceará; 2002.

24. Fontelles MJ, Simões MG, Farias SH, Fontelles RGS. (2009). Metodologia da pesquisa científica: diretrizes para a elaboração de um protocolo de pesquisa. Rev Para Med. 23 (3): 1-8.

25. Silveira DT, Córdova FP. A pesquisa científica. In: 
Gerhardt TE, Silveira DT, organizadoras. Métodos de Pesquisa. Porto Alegre: Editora da UFRGS; 2009. p. 31-42.

26. Gerhardt TE. A construção da pesquisa. In: Gerhardt TE, Silveira DT, organizadoras. Métodos de Pesquisa. Porto Alegre: Editora da UFRGS; 2009. p. 43-64.

27. Diclemente CC, Carbonari JP, Montgomery RPG, Hughes SO. (1994). The Alcohol Abstinence Self-Efficacy Scale. J stud alcohol. [internet] 1994 [acesso em 2019 Fev 15]; 55, 141-148. Disponível em: https:// www.jsad.com/doi/10.15288/jsa.1994.55.141 doi: 10.15288/jsa.1994.55.141

28. Brasil. Ministério da Saúde. Resolução $\mathrm{n}^{\circ}$ 466, de 12 de dezembro de 2012. Aprova diretrizes e normas regulamentadoras de pesquisas envolvendo seres humanos. [internet] 2012 [acesso em $2019 \mathrm{Fev} 11$ ]. Disponível em: http://bvsms.saude.gov.br/bvs/saudelegis/cns/2013/res0466_12_12_2012.html

29. Litt MD, Kadden RM, Petry NM. Behavioral treatment for marijuana dependence: Randomized trial of contingency management and self-efficacy enhancement. Addict behav. [internet] 2013 [acesso em 2019 Fev 15]; 38(3), 1764-1775. Disponível em: https://www.sciencedirect.com/science/article/abs/pii/ S030646031200305X?via\%3Dihub doi: 10.1016/j. addbeh.2012.08.011

30. Rose GL, Skelly JM, Badger GJ, Naylor MR, Helzer JE. Interactive Voice Response for relapse prevention following cognitive behavioral therapy for alcohol use disorders: A pilot study. Psychol Serv. [internet] 2012 [acesso em 2019 Fev 15]; 9(2), 174-184. Disponível em: https://psycnet.apa.org/doiLanding?doi $=10.1037 \% 2 \mathrm{Fa} 0027606$ doi: $10.1037 / \mathrm{a} 0027606$

31. Coelho LRM. Habilidades sociais e de enfrentamento no tratamento de usuários de crack [tese]. Porto Alegre: Pontifícia Universidade Católica do Rio Grande do Sul, Faculdade de Psicologia; 2016. p. 62.

32. Tenhula WN, Bennett ME, Kinnaman JES. Behavioral treatment of substance abuse in schizophrenia. J clin psychol. [internet] 2009 [acesso em 2019 Fev 15];
65(8): 831-841. Disponível em: https://onlinelibrary. wiley.com/doi/abs/10.1002/jclp.20613 doi: https:// doi.org/10.1002/jclp.20613

33. Melo MC. Sentidos construídos sobre a internação em Comunidades Terapêuticas com pessoas em tratamento por uso de drogas [dissertação]. Ribeirão Preto: Universidade de São Paulo, Faculdade de Filosofia, Ciências e Letras; 2016. 171 p.

34. Santos MPG. Comunidades Terapêuticas e a disputa entre modelos de atenção e cuidado a usuários de drogas. Boletim de Análise Político-Institucional 2018; (18): 80-9.

35. Araújo RB, Castro MG, Oliveira MS, Pedroso RS. Estratégias de coping para o manejo do craving em dependentes de tabaco. Rev bras psiquiatr. 2009; 31(2): 89-94.

36. Araújo RB, Pansard M, Boeira BU, Rocha NS. As estratégias de coping para o manejo da fissura de dependentes de crack. Clin biomed res. 2010; 30(1): 36-42.

37. Dolan SL, Martin RA, Rohsenow DJ. Self-efficacy for cocaine abstinence: Pretreatment correlates and relationship to outcomes. Addict behav. [internet] 2008 [acesso em 2019 Fev 15]; 33(5): 675-688. Disponível em: https://www.sciencedirect.com/science/ article/abs/pii/S0306460307003322?via\%3Dihub doi: 10.1016/j.addbeh.2007.12.001

38. Martin RA, Rohsenow DJ, MacKinnon SV, Abrams DA, Monti PM. Correlates of motivation to quit smoking among alcohol dependent patients in residential treatment. Drug alcohol depend. [internet] 2006 [acesso em $2019 \mathrm{Fev}$ 15]; 83(1): 73-78. Disponível em: https://jhu.pure.elsevier.com/en/ publications/correlates-of-motivation-to-quit-smoking-among-alcohol-dependent--3 doi: 10.1016/j. drugalcdep.2005.10.013

39. Sá LGC, Del Prette ZAP. Habilidades Sociais como Preditoras do Envolvimento com Álcool e Outras Drogas: Um estudo exploratório. Interação Psicol. [internet] 2014 [acesso em 2019 Fev 15]; 18(2): 167- 
178. Disponível em: https://revistas.ufpr.br/psicoloRecebido em: 16/02/2019 gia/article/view/30660 doi: 10.5380/psi.v18i2.30660

40. Kessler FHP, Terra BM, Faller S, Stolf AR, Peuker $\mathrm{AC}$, Benzano D, et al. Crack users show high rates of antisocial personality disorder, engagement in illegal activities and other psychosocial problems. The American Journal of Addictions. [internet] 2012 [acesso em 2019 Fev 15]; 21(4): 370-380. Disponível em: https:/onlinelibrary.wiley.com/doi/ful1/10.1111/j.1521-0391.2012.00245.x doi: 10.1111/j. 1521-0391.2012.00245.x

41. Felipe AOB, Carvalho AMP, Andrade CUB. Espiritualidade e religião como protetores ao uso de drogas em adolescente. SMAD, Rev eletrônica saúde mental alcool drog. [internet] 2015 [acesso em 2019 Fev 15]; 11 (1): 49-58. Disponível em: http://pepsic.bvsalud.org/scielo.php?pi$\mathrm{d}=$ S1806-69762015000100008\&script $=$ sci_arttext\&tlng =pt doi: 10.11606/issn.1806-6976.v11i1p49-5

42. Szupszynnki KPR, Oliveira MS. Adaptação brasileira da University of Rhode Island Change Assessmente (URICA) para usuários de substâncias ilícitas. Psico-USF. [internet] 2008 [acesso em 2019 Fev 15]; 13(1): 31-39. Disponível em: http://www.scielo.br/scielo. php? pid $=S 1413=82712008000100005-\&$ script sci_abstract\&tlng $=$ pt $\quad$ doi: $\quad 10.1590 / \$ 1413-$ 82712008000100005

43. McLellan A, Luborsky L, Woody G, OBrien C. An improved diagnostic evaluation instrument for substance abuse patients. J nerv ment dis. [internet] 1980 [acesso em 2019 Fev 15]; 168(1): 26-33. Disponível em: https://insights.ovid.com/ crossref?an $=00005053-198001000-00006 \quad$ doi: 10.1097/00005053-198001000-00006

44. Carmo JT, Pueyo AA. A adaptação ao português do Fagerström test for nicotine dependence (FTND) para avaliar a dependência e tolerância à nicotina em fumantes brasileiros. Rev Bras Educ Méd. 2002; 59: $73-80$ 